\title{
A systemic inflammation response index (SIRI) correlates with survival and predicts oncological outcome in resected pancreatic cancer following neo-adjuvant chemotherapy
}

\author{
$\underline{\text { Ji Su KIM }}{ }^{1,2}$, Chang Moo KANG ${ }^{* 1,2}$ \\ 'Department of Hepatobiliary and Pancreatic Surgery, Yonsei University College of Medicine, Seoul, Korea \\ ${ }^{2}$ Pancreatic Cancer Center, Yonsei Cancer Center, Severance Hospital, Seoul, Korea
}

Introduction: The systemic inflammation response index (SIRI, neutrophil x monocyte/lymphocyte) is emerged in predicting oncological prognosis in various type of cancer. We investigated to examine whether SIRI is significant as a prognostic factor even in neo-adjuvant settings, and whether changes in SIRI values after neo-adjuvant chemotherapy are related to prognosis.

Methods: Medical records were investigated retrospectively about patients underwent pancreatic surgery following neo-adjuvant chemotherapy for pancreatic cancer, from 2006 to 2019. All 160 patients were investigated. In order to find the meaningful SIRI values and their changes, values of division and as well SIRI pre-neoadjuvant and SIRI post-neoadjuvant were calculated. Among calculated SIRI values, statistically significant SIRI values were found using statistical techniques to determine the effect on the survival rate.

Results: The value of SIRI post-neoadjuvant $<0.8710$ affected the prognosis of patients in the univariate ${ }^{\star}$ and multivariate analysis ${ }^{* *}$ $\left(p\right.$-value ${ }^{\star}=0.0317, p$-value $\left.{ }^{*}=0.0066\right)$. The value of $\Delta$ SIRI $<0.9516$ affected the prognosis of patients in the univariate ${ }^{*}$ and multivariate analysis ${ }^{\star *}\left(p-\right.$ value $^{\star}=0.0380, p$-value $\left.e^{\star *}=0.00462\right)$. In Kaplan-Meier curve, the disease free survival was significantly different between the group of SIRI post-neoadjuvant $<0.8710$ and SIRI post-neoadjuvant $\geq 0.8710(p=0.0303)$. The overall survival was significantly different between the group of SIRI post-neoadjuvant/pre-neoadjuvant $<0.9516$ and SIRI post-neoadjuvant/pre-neoadjuvant $\geq 0.9516(p=0.0368)$.

Conclusions: An SIRI can be used to predict the survival of patients with pancreatic cancer after pancreatic resection following neo-adjuvant chemotherapy. 